\title{
An unusual cause of hemosuccus pancreaticus
} diagnosed by endoscopic ultrasonography: splenic arterial fistula due to a pancreatic stent (with video)

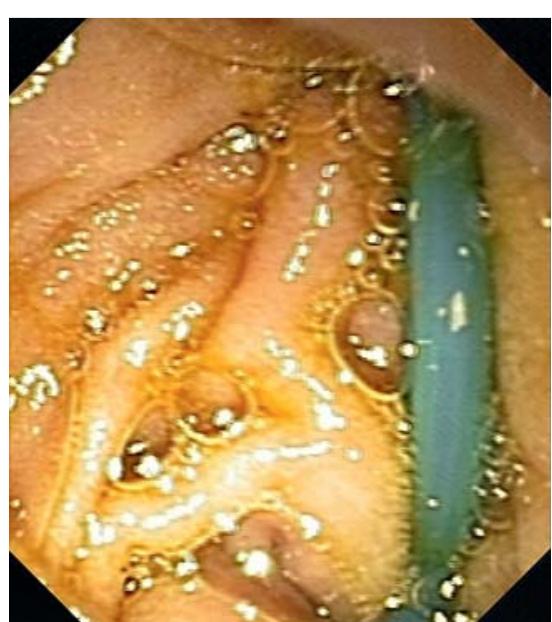

Fig. 1 Presence of the pancreatic plastic stent in the second duodenum.

We report the case of a 54-year-old woman recently diagnosed with severe acute biliary pancreatitis requiring surgical necrosectomy and caudal pancreatectomy 1 month later. After surgery the patient developed an internal pancreatic fistula which was treated with octreotide (Sandostatin) and percutaneous drainage. Two months later she was diagnosed with a large pancreatic pseudocyst communicating with the pancreatic duct. A transpapillary plastic stent was placed with favorable outcome. After 2 months intermittent rectal bleeding occurred.

The patient was hospitalized 3 weeks after the onset of the intermittent rectal bleeding, with normal blood pressure and severe anemia (hemoglobin $6.9 \mathrm{~g} / \mathrm{dL}$ ). Upper gastrointestinal endoscopy showed the presence of the pancreatic stent and blood in the second part of the duodenum, coming out of the papilla ( $\bullet$ Fig. 1). Because there was no possibility of emergent CT or MRI examination, endoscopic ultrasonography (EUS) was performed and showed the plastic pancreatic stent in the pancreatic duct with the distal end in the splenic artery ( Figs. 2-5, - Video 1).

Urgent laparotomy was performed and confirmed that the splenic artery wall had been penetrated by the pancreatic stent; there was a large old hematoma around it. Distal pancreatectomy with double ligation of the splenic artery and

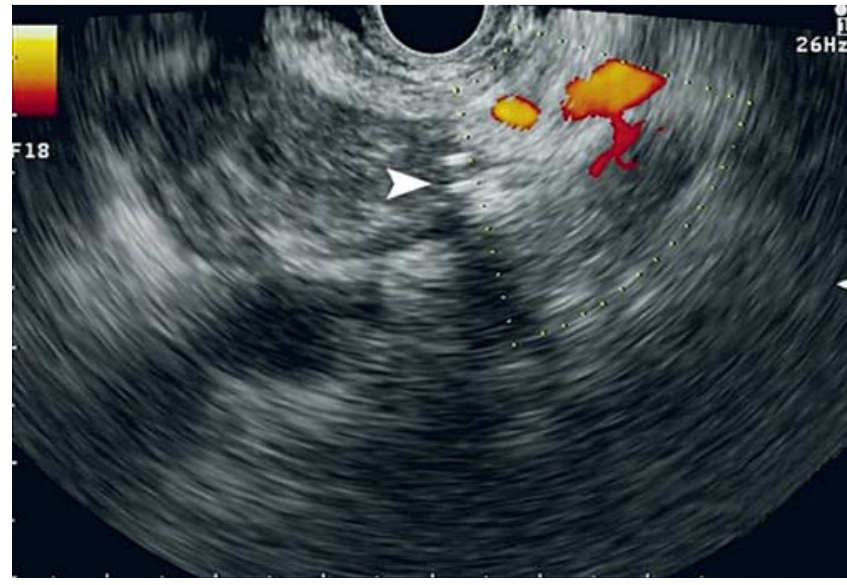

Fig. 2 Stent visible inside a hypoechoic pancreatic structure (arrowhead).

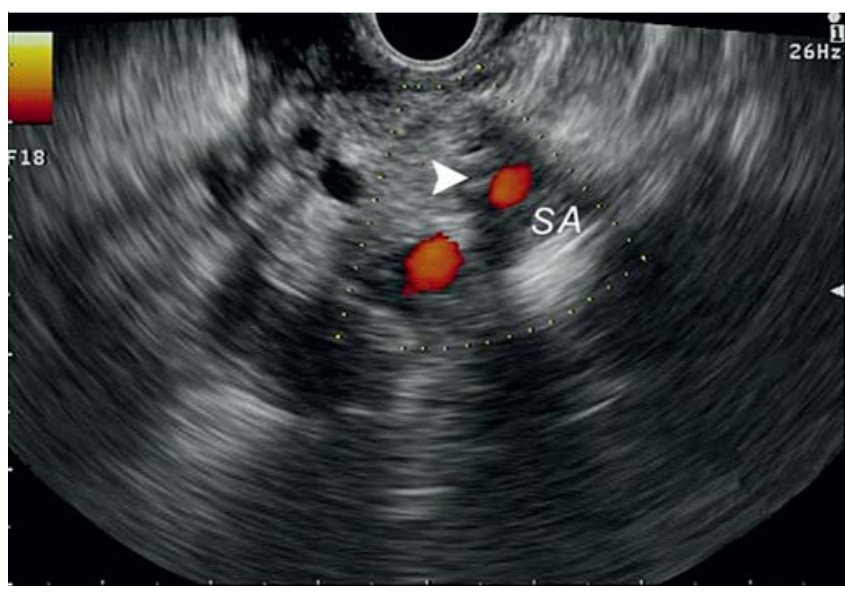

Fig. 3 Distal end of the stent (arrowhead) visible inside the splenic artery (SA).

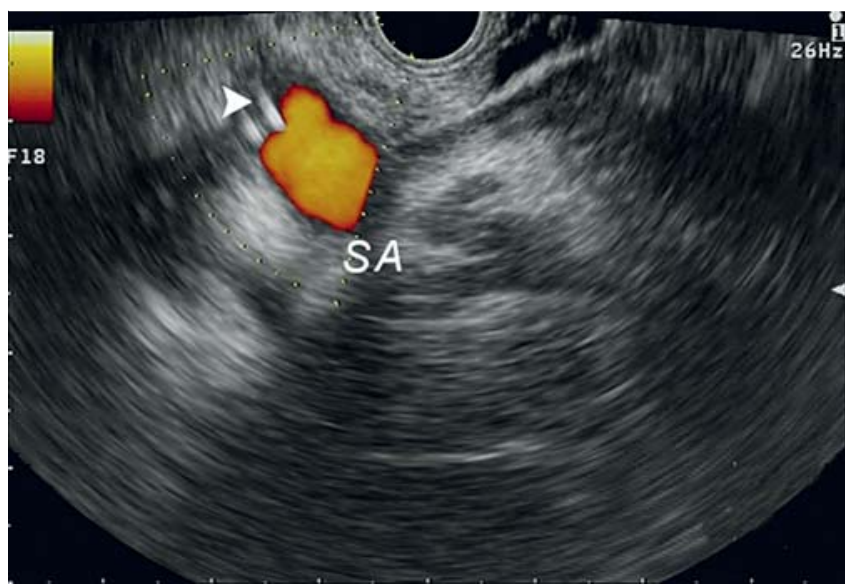

Fig. 4 Distal end of the stent (arrowhead) visible inside the splenic artery (SA), with the left adrenal gland nearby.

splenectomy were successfully performed with favorable outcome immediately and 3 months later.

Hemosuccus pancreaticus is defined as papillary bleeding from the pancreatic duct [1]. The main causes are divided into those of pancreatic origin (rupture into the pancreatic duct of a pseudoaneurysm associated with acute or chronic pancreatitis, arteriovenous malformations, pan- 


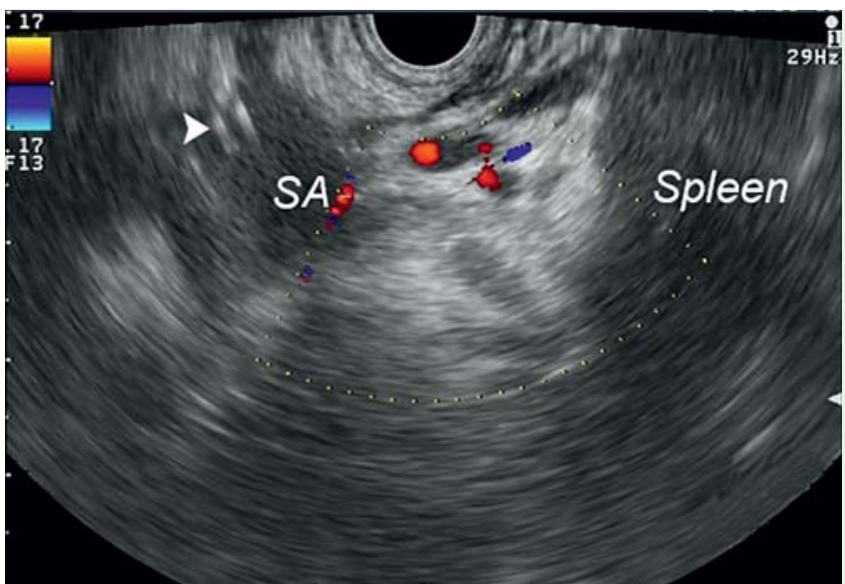

Fig. 5 Distal end of the stent (arrow) visible inside the splenic artery $(\mathrm{SA})$, with the collateral circulation in the splenic hilum nearby. creatolithiasis, tumors or bleeding after EUS fine-needle aspiration for cystic lesions) and those of extrapancreatic origin (rupture of a true splenic aneurysm) [2,3]. This is the first report of a splenic arterial fistula caused by penetration of a pancreatic stent after resolution of the pancreatic collection, diagnosed by EUS, and treat-

\section{Video 1}

During endoscopic ultrasonography the plastic pancreatic stent in the pancreatic duct is visible as a parallel hyperechoic structure, crossing a hypoechoic structure (hematoma) and with the distal end in the splenic artery (vascular hypoechoic structure with Doppler and power Doppler signal). ed surgically. Other ways of treatment could be transarterial catheter embolization with implantation of a coil or prosthesis, local injection of Histoacryl, or pancreatic resection $[4,5]$.

\section{Competing interests: None}

$$
\text { Endoscopy_UCTN_Code_CPL_1AK_2AJ }
$$

A. Seicean ${ }^{1}$, R. Stan-luga ${ }^{1}$, D. Munteanu ${ }^{2}$

1 Third Medical Clinic, Iuliu Hatieganu University of Medicine and Pharmacy, Cluj-Napoca, Romania

2 Third Surgical Clinic, Iuliu Hatieganu University of Medicine and Pharmacy, Cluj-Napoca, Romania

\section{References}

1 Sandblom P. Gastrointestinal hemorrhage through the pancreatic duct. Ann Surg 1970; 171: $61-66$

2 Massani M, Bridda A, Caratozzolo E et al. Hemosuccus pancreaticus due to primary splenic artery aneurysm: a diagnostic and therapeutic challenge. JOP 2009; 10: $48-52$

3 Cheruvattath R, Diehl DL. Hemosuccus pancreaticus after EUS-FNA of a pancreatic tail cyst. Gastrointest Endosc 2009; 70: 817

4 Tabrizian P, Reiter BC, Heimann TM. Multimodality treatment for hemosuccus pancreaticus. Am J Gastroenterol 2009; 104: 1060

5 Will U, Mueller AK, Grote R et al. "Hemosuccus pancreaticus" - primarily ultrasoundguided successful intervention using transcutaneous fibrin glue application and histoacryl injection. Ultraschall Med 2008; 29 : $260-263$

\section{Bibliography}

Dol $10.1055 / \mathrm{s}-0030-1255619$

Endoscopy 2010; 42: E239-E240

(c) Georg Thieme Verlag KG Stuttgart · New York . ISSN 0013-726X

\section{Corresponding author}

\section{A. Seicean, MD, PhD}

Clinica Medicală III

UMF "Iuliu Hațieganu"

Str. Croitorilor Nr. 19-21

Cluj-Napoca

Romania

andradaseicean@yahoo.com 